\title{
Imprinted Spiral Structures as Neutron Polarizers
}

'W. Lohstroh, ${ }^{2}$ G.P. Felcher, ${ }^{2}$ R. Goyette, ${ }^{1} \mathrm{M}$. Munzenberg, and 'W. Felsch

${ }^{2}$ I. Physikalisches Institut Universitat Gottingen, 37073 Gottinggen, Germany

${ }^{2}$ Argonne National Laboratory, Argonne, Illinois 60439 .

The submitted manuscript has been created The subnited Argonne National Laboratory ("Argonne") Argonne National Laborat-109-ENG-38 with under Contract No. W-31-109-ENG-38 W.S. the U.S. Department of Energy. The U.S. Government retains for itself, and others acting on its behalf, a paid-up, nonexclusive, irrevocable worldwide license in said article to reproduce, prepare derivative works, distribute copies to the public, and pertorm publicly and display publicly, by or on behalf of the Govemment.

To be published in Physica B

Work at Argonne was supported by the U.S. Department of Energy, BES-Material Sciences, under Contract No. W-31-109-ENG-38. Work in Gottingen was supported by the Deutsche Forschungsgemeinschaft within SFB 345. 


\section{DISCLAIMER}

This report was prepared as an account of work sponsored by an agency of the United States Government. Neither the United States Government nor any agency thereof, nor any of their employees, make any warranty, express or implied, or assumes any legal liability or responsibility for the accuracy, completeness, or usefulness of any information, apparatus, product, or process disclosed, or represents that its use would not infringe privately owned rights. Reference herein to any specific commercial product, process, or service by trade name, trademark, manufacturer, or otherwise does not necessarily constitute or imply its endorsement, recommendation, or favoring by the United States Government or any agency thereof. The views and opinions of authors expressed herein do not necessarily state or reflect those of the United States Government or any agency thereof. 


\section{DISCLAIMER}

Portions of this document may be illegible in electronic image products. Images are produced from the best available original document. 


\title{
IMIPRINTED SPIRAL STRUCTURES AS NEUTRON POLARIZERS
}

\author{
W. Lohstroh ${ }^{a}$, G. P. Felcher ${ }^{b}$, R. Goyette ${ }^{b}$, M. Münzenberg ${ }^{a}$, and W. Felsch \\ ${ }^{a}$ I. Physikalisches Institut Universität Göttingen, 37073 Göttingen, Germany, \\ 'Argonne National Laboratory, Argonne, II 60439, USA
}

Neutron diffraction from magnetic spiral structures is governed by strong selection rules for the polarization of the outgoing beam. When the sample is entirely of one chirality - for instance a right handed spiral - the neutrons diffracted by some Bragg reflections are fully polarized. While the scattering theory has been formulated long ago, attempts to controllably modify the population of left handed and right handed spiral domains in natural magnetic structures (which for instance occur in some rare earth metals) have been largely unsuccessful. In contrast, we have been able to imprint helical magnetic structures in $\mathrm{La} / \mathrm{Fe}$ multilayers (each layer approximately $30 \AA$ Ahick) simply by rotating the growing sample in a weak external field (3Oe). A first estimate is given of the efficiency of these multilayers as polarizers of neutron beams.

PACs: $75.25 .+z, 75.70 .-\mathrm{i}$

Keywords: neutron reflectometry, neutron polarizer

Corresponding author: W. Lohstroh

I. Physikalisches Institut Universität Göttingen

Bunsenstr. 9

37073 Göttingen, Germany

Phone: +49-55I-397605

Fax: +49-551-394493

wlohstr@gwdg.de 
The search for efficient neutron polarizers has engaged researchers for many years. The first devices that made possible the practical use of polarized neutrons were ferromagnetic single crystals, aligned to diffract neutrons from a selected. Bragg reflection. The diffracted intensity for neutrons with polarity either up or down with respect to the magnetization depends on the magnetic and nuclear structural factors, and is proportional to:

$$
I^{ \pm}=\left|F_{N}(q) \pm F_{M}(q)\right|^{2}
$$

as a function of the momentum transfer $\mathbf{q}$. Hence the diffracted neutrons are fully polarized if $\left|F_{N}(q)\right|=\left|F_{M}(q)\right|$. The strong constraint restricts the choice of neutron polarizers to very few crystals, and for all of them the polarization efficiency parameter $\mathrm{E}=\dot{\mathrm{P}} \times \mathrm{T}(\mathrm{P}=$ polarization, $\mathrm{T}=$ : transmission) is substantially less than the ideal value, 0.5 . More recently optical devices have been constructed for neutrons of intermediate and long wavelengths. The optical devices are basically magnetized mirrors which utilize the splitting of the total reflection angle for neutrons polarized parallel and opposite to the magnetization. Simple mirrors, however, severely restrict the size and divergence of the incoming beam, because the critical angles for total reflection are only of the order of one milliradiant for $1 \AA$ neutrons. Only after the recent improvements of the techniques of deposition more efficient optical devices have been constructed. The supermirrors [1] consist of a generalized multilayer sequence which effectively reflects $1 \AA$ neutrons to angles up to 5 milliradiants. As in the case of diffracting crystals, to entirely suppress the scattering of the undesired spin state it is required that $\left|F_{N}(q)\right|=\left|F_{M}(q)\right|$.

An entirely different solution to the problem has been proposed since the early sixties. This takes advantage of the diffraction properties peculiar to magnetic spiral structures. Spirals give rise to entirely magnetic Bragg reflections. Indicating with $\mathrm{Q}$ the momenta of the structural reflections, magnetic diffraction peaks (usually called satellites) appear around $Q$ at the reciprocal space positions: 


$$
\mathbf{q}=\mathbf{Q} \pm \tau
$$

where $\tau=2 \pi / \Lambda$, the magnetic periodicity, and its direction depends on the chirality, being opposite for right handed and left handed spirals. Conventionally, the intensities associated with

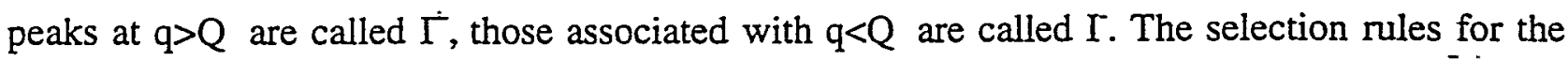
polarization of the diffracted beam take a form particularly simple when $Q$ and $\tau$ are collinear. In this case

$$
I^{ \pm} \div 1 \pm(\text { P.q)(q. } \tau)
$$

The neutron polarization can be defined with respect to a guide magnetic field $\mathbf{H}$ which we may choose to be parallel to $q$. For neutrons in the polarization state $P . q=1$ a finite diffraction peak $\mathrm{I}^{+}$: takes place only for a right handed spiral $(q . \tau=1)$. Similarly, neutrons in the polarization state P.q $=-1$ are diffracted only at $I^{*}$ by a right handed spiral. For a left handed spiral the same selection rules are valid, but for reversed states of the neutron polarization.

Magnetic spiral structures occur in several rare earth metals like Tb, Ho and Er. However the first material whose sample was found to have a preferential chirality was $\mathrm{MnP}$ [2]. Extensive investigations of the size and position of domains of opposite chirality for several spiral structures have taken place by neutron topography [3]. However, repeated attempts to modify controllably the domain population (for instance, by thermal cycling) were mostly unsuccessful $[4,5]$. Only in non-centrosymmetric $\mathrm{MnSi}$ the onset of spirals with one chirality have been theoretically predicted and experimentally observed [6]. Unfortunately $\mathrm{MnSi}$, with a $29 \mathrm{~K}$ Neél temperature and an ordered moment of $0.4 \mu_{\mathrm{B}} / \mathrm{Mn}$-atom, is hardly the best candidate for a practical polarizer.

Helical structures have also been found in artificial multilayers and superlattices. The use of such structures as neutron polarizers has been proposed by Majkrzak [7]. Here again. however, the problem is to obtain samples consisting of domains of one chirality only. Conventionally, the 
magnetic structure of metallic multilayer systems is determined by the exchange energy between successive ferromagnetic layers separated by spacers. In contrast, recently we have shown that in $\mathrm{La} / \mathrm{Fe}$ multilayers this interaction is so weak that the magnetic structure can be imprinted simply by choosing an appropriate method of preparation [8].

The La/Fe samples were grown by ion beam sputtering in an UHV chamber (base pressure below $5 \cdot 10^{-10} \mathrm{mbar}$ ) on $\mathrm{Si}(100)$ substrates covered with a $40 \AA \mathrm{Cr}$ buffer. The samples have a nominal composition of $[\mathrm{La} 32 \AA / \mathrm{Fe} 30 \AA] \times 32$, and were covered with $100 \AA \mathrm{Pd}$ to provide protection against oxidation. During the deposition the substrate was exposed to a small magnetic field ( 3 Oe) while continuously rotated -originally to ensure homogenous layer growth. The : magnetic structure was determined by polarized neutron reflectometry at the POSY 1 instrument of the Intense Pulsed Neutron Source in Argonne [9]. Fig 1. shows the neutron spectrum of a sample [La $32 \AA / F e ~ 30 \AA] \times 32$ prior to any exposure to a magnetic field. The spin dependence of the reflectivity indicates that the sample exhibits a single chirality over the entire surface area amounting to several $\mathrm{cm}^{2}$. Further experiments proved that the magnetic structure was indeed imprinted during preparation: for instance, by rotating the samples in the opposite direction, the chirality of the spiral was reversed. The magnetic structure were found to be stable in time. However they were irreversibly erased by exposure to magnetic fields exceeding 90 Oe.

Can imprinted spiral structures be used as efficient new neutron polarizers? The polarization efficiency parameter here can be expressed by $E=P \times R$, where $P$ is the polarization and $R$ the reflectivity. The reflectivity of the $0^{+}$reflection is rather low, but can be improved. The La/Fe layers are well formed, as proven by X-ray diffraction measurements, showing up to seven satellite peaks due to the bilayer periodicity in the small angle spectra. However not well controlled up to now has been the imprinting magnetic field: this was not necessarily homogeneous over the sample area, and the sample's rotational speed was not optimized. The 
polarization of $0^{+}$is already rather good. It has to be reminded that in reflectivity at the $0^{+}$ position there is still a contribution due to the nuclear part of the potential, but has decayed from the total reflection edge with $\mathrm{a} \mathrm{q}^{-4}$ law.

For the-sample shown in Fig. 1, the periodicity amounts to 3.3 times the bilayer period, and as a result the $0^{+}$reflection appears at a value of $\mathrm{q}$ that in practical devices is already well covered by supermirrors. Such value cannot be changed by a large amount. The periodicity of the spiral should be longer than two bilayer spacings - the limit at which the multilayer is simply antiferromagnetic. On the other hand, the multilayers become unstable if the thickness of either the $\mathrm{La}$ or the Fe layers is reduced below $25 \AA$. The $1^{-}$or $1^{+}$satellites reflect neutrons in geometrical conditions that are intermediate between those of the supermirrors and the single crystal polarizers. It is true that their polarization efficiency - as obtained in this serendipitous experiment - is rather low, but we cannot foresee any inherent mechanism that would prevent a dramatic enhancement of its value.

\section{Acknowledgments}

Work in Göttingen was supported by the Deutsche Forschungsgemeinschaft within SFB 345. Work at Argonne was supported by US department of energy, Basic Energy Science, Material Science contract \#W-31-109-ENG-38. 


\section{References:}

[1] Thin-Film Neutron Optical Devices: Mirrors, Supermirrors, Multilayer, Monochromators, Polarizers, and Beam Guides, C.F. Majkrzak and A.M. Saxena, eds. SPIE Proceedings, vol. 983, SPIE Bellingham, WA (1988)

[2] G.P. Felcher, G.H. Lander and T.O. Brun, Journal de Physique 32, C1-575 (1971)

[3] J. Baruchel, M. Schlenker and S.B. Palmer, Nondestr. Test. Eval. 5, 349 (1990)

[4] J. Baruchel, S.B. Palmer and C. Patterson, Journal de Physique 49, C8-1893 (1988)

[5] J. Sandonis, A. Pearce, J. Baruchel, K. Parlinski and S.B. Palmer, J.M.M.M. 104-107, 345 (1992)

[6] G. Shirane, R. Cowley, C.F. Majkrak, J.B. Sokoloff, B. Pagonis, C.H. Perry, and Y. Ishikawa, Phys. Rev. B 28, 6251 (1983)

[7] C.F. Majkrak, Physica B 156 \& 157, 619 (1989)

[8] G.P. Felcher, W. Lohstroh, H. Fritzsche, M. Münzenberg, H. Maletta, and W. Felsch, Appl. Phys. Lett. 72, 2894 (1998)

[9] G.P. Felcher, R.O. Hilleke, R.K. Crawford, J. Haumann, R. Kleb, and G. Ostrowski, Rev. Sci. Instruments. 58, 609 (1987) 


\section{Figure Caption:}

Fig. 1: Polarized neutron reflectivity of $[\mathrm{La} 32 \AA$ /Fe $30 \AA] \times 32$. The solid and open symbols show the $\mathrm{R}^{+}$and $\mathrm{R}^{-}$reflectivity, respectively. The magnetic satellites $\mathrm{I}^{+}$occur solely in the $\mathrm{R}^{+}$intensity whereas the $\Gamma$ satellites show up only for the neutron beam polarized in the opposite direction. 


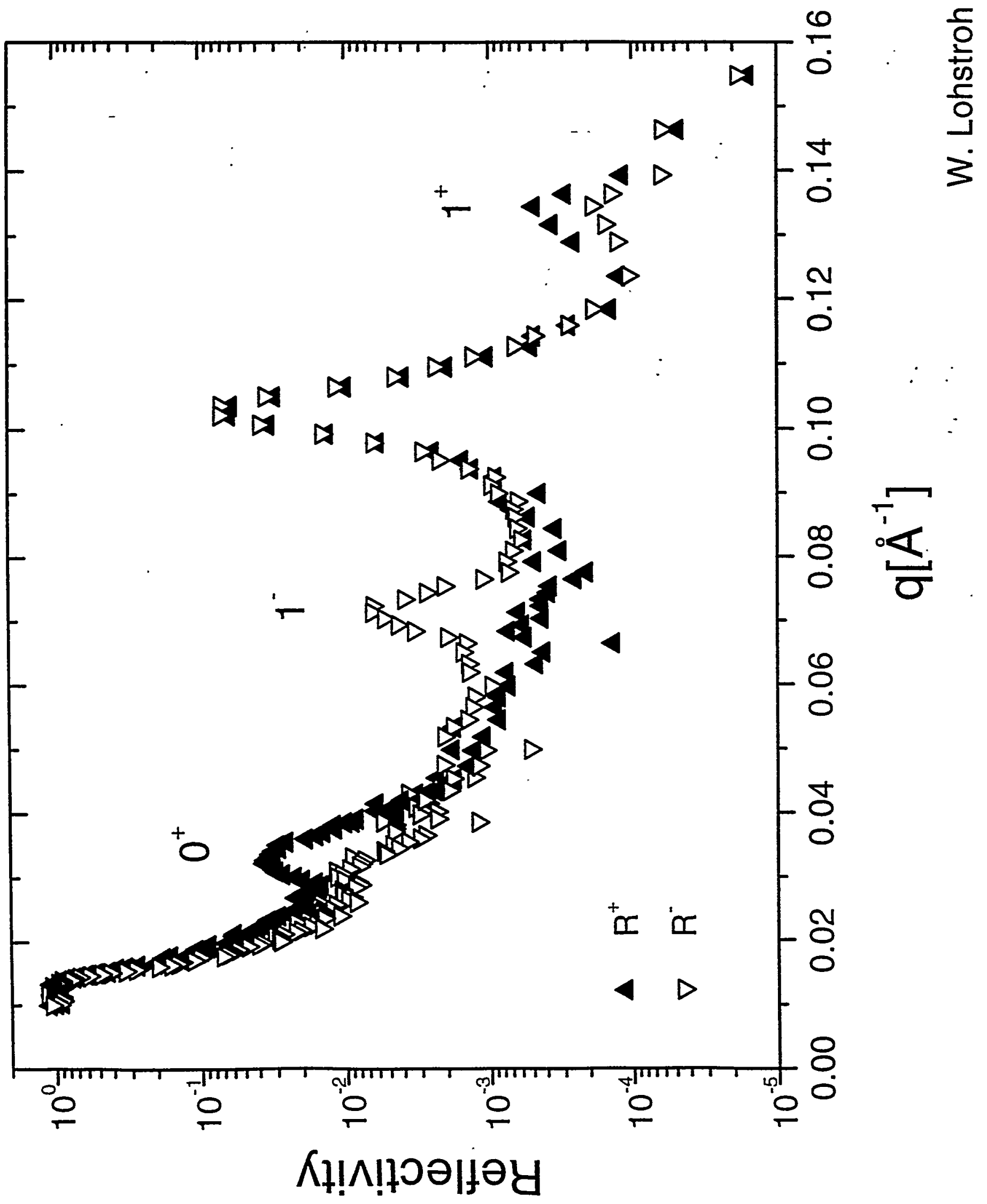

